\title{
Measurement of stress waves induced by a laser pulse
}

\author{
Joanna Radziejewska ${ }^{1}$, Jan Marczak ${ }^{2}$, Antoni Rycyk ${ }^{2}$, Karol Jach $^{2}$, Antoni Sarzyński² ${ }^{* 2}$ and Marek Strzelec ${ }^{2}$ \\ ${ }^{1}$ Institute of Fundamental Technological Research, Polish Academy of Sciences, 5B Pawińskiego Street; 02-106 \\ Warszawa, Poland \\ ${ }^{2}$ Institute of Optoelectronics, Military University of Technology, 2 Gen. S. Kaliskiego Street, 00-908 Warszawa, \\ Poland
}

Received August 28, 2015; accepted October 07, 2015; published December 31, 2015

\begin{abstract}
This letter describes modelling and experimental results of low and high pressure shock waves (of the order of 100 and 1000 bar, respectively) induced by pulse laser radiation. Measurements were performed using piezoelectric polymer PVDF sensors. The results of numerical calculations and their comparison with an experiment have been posted. Measured and calculated results have been used in estimation of the temporary course of signals from a fibre VISAR interferometer which measured the movement velocity of a steel plate driven by the analysed shock waves. The description is supplemented with the results of magnitude measurements of surface plastic deformation and material microstructure of samples.
\end{abstract}

The behaviour of materials and layers under high strain rates significantly differs from the behaviour in static conditions, and is difficult to analyse using methods [1] hitherto applied. Very large deformation rates occur during friction, mechanical treatment and exploitation of many materials and technical components. Determined mechanical dynamic properties of materials and composites, such as dynamic hardness, layer adhesion to the substrate, dynamic yield strength, residual stresses of layers, allow proper selection of materials and layers for specific applications. Also, the detection of defects and material deformations that are created during their use becomes possible. Intense, short-term laser pulses are an ideal tool to induce short pressure pulses with large amplitude in materials, and to apply them in the investigation of deformation processes and destruction of solids and layers at rates in the range of $10^{6}-10^{9} \mathrm{~s}^{-1}$ [2]. This is the main aim of the described below simulation and experimental analyses of the parameters of laser pulse interaction with materials and its influence on the values of shock wave pressures.

A typical experimental stand is shown in Fig. 1. A laser beam (1) falls through the transparent inert layer (2) onto the absorption-ablation-protective layer (3), where it is absorbed and creates high pressure plasma, which in turn induces a shock wave (4). The propagation of the shock wave causes plastic deformations in the processed layer (5). The piezoelectric PVDF sensor (6) creates a charge which is proportional to the transient value of pressure in

\footnotetext{
${ }^{*}$ E-mail: antoni.sarzynski@wat.edu.pl
}

the piezoelectric sensor. The shock wave propagates through the substrate (7) and finally reaches the analysed layer (8). The movement velocity of the sample external free surface can be measured by means of a fibre VISAR (Velocity Interferometer System for Any Reflector) interferometer.

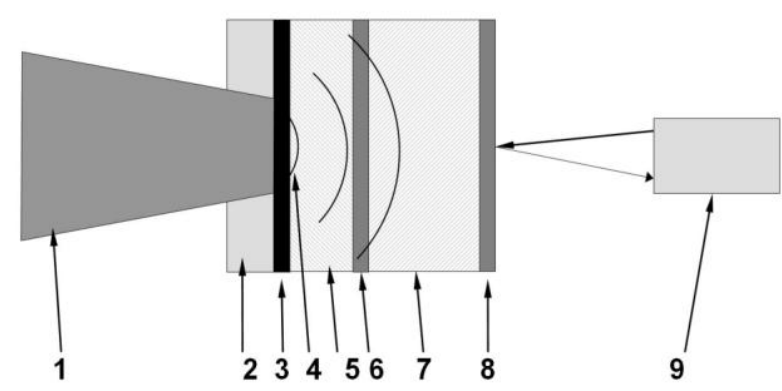

Fig. 1. Experimental scheme for testing adhesion of thin layers to the substrate: 1 - laser pulse; 2 - inert layer (glass); 3 -absorption layer; 4 - stress wave front; 5 - steel plate; 6 - unit of PVDF piezoelectric sensor layers; 7 - substrate; 8 - analysed thin-film coating; 9 - VISAR interferometer.

Numerous measurements of shock wave pressures, induced by pulse laser radiation have been performed using piezoelectric, polymer PVDF sensors. The regularity of some measurements could be easily verified, but several results have raised doubts as to the origin of the shape of a shock wave or to amplitude dispersion. Therefore, computer simulations were performed to clarify these issues. A hydrodynamic code, similar to the one used in [3] was developed to simulate the radiation absorption, pressure, temperature and density of expanding plasma as well as the excitation and propagation of shock waves. Equations, in the form of one-dimensional hydrodynamic equations, included radiation absorption, electron and radiative heat conduction, shock waves and ionisation losses. The equations were solved using an explicit differential method. The computer code included five layers with the following assumption and data: as an inert layer, glass 
with a thickness of $3.5 \mathrm{~mm}$ was used; as the absorption layer - graphite with a thickness of $150 \mu \mathrm{m}$; as the processed layer - a stainless steel plate with a thickness of $0.8 \mathrm{~mm}$; the fourth layer was a sensor with a thickness of $175 \mu \mathrm{m}$, and as the substrate, a commercial PVDF polymer was used, having the properties slightly different than those of the PVDF piezoelectric sensor.

The comparative results of measurements and calculation of pressures of a shock wave (amplitudes of the order of 100 and 1000 bar) are shown in Fig. 2 and Fig. 3. In all experiments, the diameter of laser beam incident on the sample was about $3 \mathrm{~mm}$, and was generally much larger than the thickness of the tested sample of $0.5 \div 1 \mathrm{~mm}$. Therefore, for the time period of $0.5 \div 1 \mu \mathrm{s}$ the approximation of a plane wave can be regarded as justified.

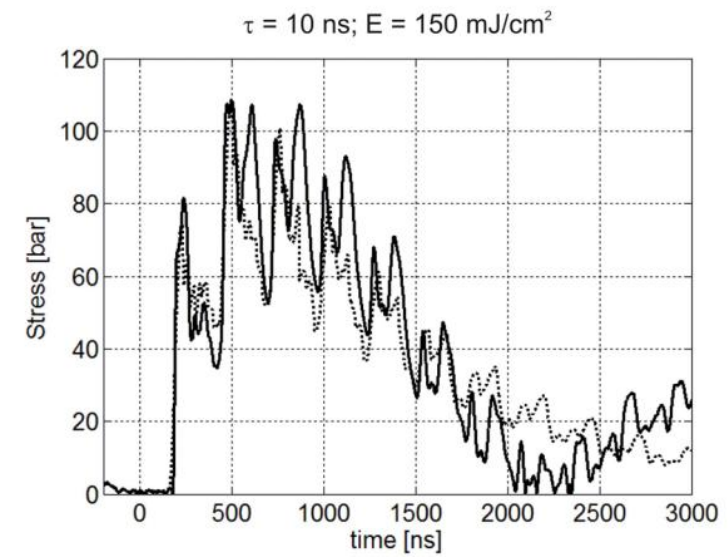

Fig. 2. Comparison of the calculated (dotted line) and experimental (solid line) profile of pressure of a low amplitude shock wave.

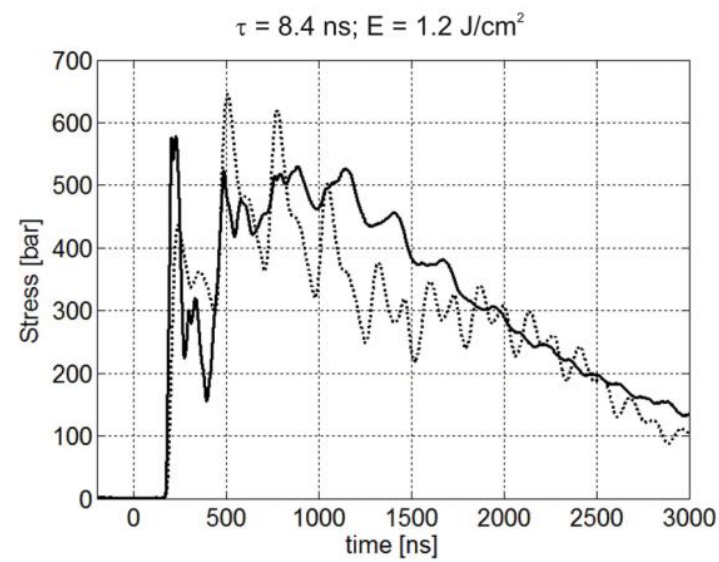

Fig. 3. Comparison of the calculated (dotted line) and experimental (solid line) profile of pressure of a high amplitude shock wave.

It should be noted that the measurement results presented in Figures 2 and 3 relate to the pressure in the interior of the PVDF sensor. The pressure of a shock wave in the investigated steel plate is nearly ten times higher.

The consistency of theory and experiment in both cases covers a time period of around 500ns. Later, the consistency of results disappears, mainly due to twodimensional effects. The stress wave for a long time circulates with the speed of sound inside the tested steel plate in the case of low amplitude (Fig. 2), which results in rhythmically repeated stress peaks every $270 \mathrm{~ns}$. In the case of a high amplitude shock wave, plastic deformations occurring in the steel plate and nonlinear phenomena cause a change of shape of the sensor signal. It substantially extends in time and its oscillating shape disappears (Fig. 3).

The results that are presented in Fig. 2 and Fig. 3 allowed verifying the correctness of conducted numerical calculations. It was therefore assumed that the program developed enables also correct estimation of free surface velocity of the analysed thin-film coating also in an easier case, without plate (5) and sensor unit (6).
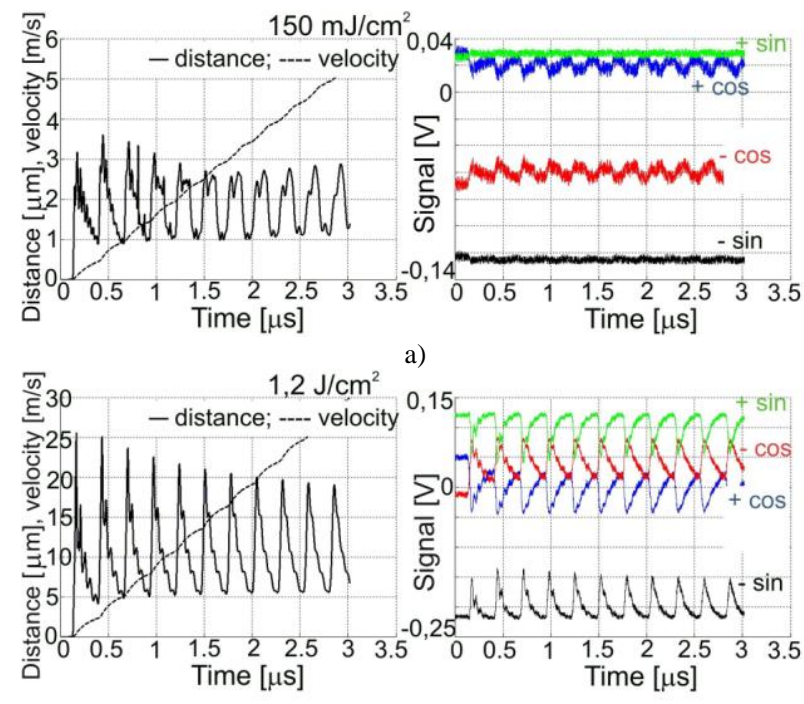

b)

Fig. 4. Estimation of velocity and displacement of a steel plate with a thickness of $0.5 \mathrm{~mm}$, driven by a laser pulse-induced stress wave (left side), and signals of the VISAR system in the case of: a) a low amplitude and b) high amplitude shock wave.

The results obtained have been used to simulate signals of the interferometric VISAR measurement system. Two beams, reflected from the analysed object, interfere with the delay of a given time period $\tau$ in this type of interferometer [4]. The system records pairs of four signals: $\pm \sin$ and $\pm \cos$. A phase delay of $\pi / 2$ should occur between $\sin / \cos$ signals, and $\pi$ between \pm signals. The signals in a real interferometer, taking into account variable values of laser beam power are described by formulas [4]: 


$$
\begin{aligned}
& C_{1}=x_{0}+A_{x} \cos (\Phi(t)), \\
& C_{2}=x_{0}+A_{x} \cos (\Phi(t)+\pi), \\
& S_{1}=y_{0}+A_{y} \sin (\Phi(t)+\varepsilon), \\
& S_{2}=y_{0}+A_{y} \sin (\Phi(t)+\pi+\varepsilon), \\
& \Phi(t)=\beta_{0}+4 \pi V(t) \tau / \lambda .
\end{aligned}
$$

where: $V$ - velocity; $\tau$ - time delay; $\lambda$ - wavelength; $\beta_{0}$ constant phase delay; $x_{0}, y_{0}-$ constant components; $A_{x, y}$ variable components; $t$ - time, $\varepsilon$ - phase mismatch of sin/cos signals.

Figure 4 shows the results of calculations of displacement and velocity of a surface driven by a low and high amplitude shock wave, placed together with estimated signals of the VISAR system.

It should be stressed that even in the case of high amplitude (Figure 4b), the shock wave is a weak one so its velocity of propagation is only slightly higher than the speed of sound. Knowing the velocity of the rear surface of the sample (Fig. 4b) it is possible to estimate the peak pressure of the shock wave from the formula $P=\rho v c-$ it is around 5000 bar $\left(\rho=8 \mathrm{~g} / \mathrm{cm}^{3} ; c=5 \cdot 10^{5} \mathrm{~cm} / \mathrm{s} ; v=12.5 \mathrm{~m} / \mathrm{s}-\right.$ because the mass velocity doubles in reflection from a free surface).

It can be seen that even in the case of a high amplitude shock wave (Fig. 4b), the surface displacement velocity does not exceed $50 \mathrm{~m} / \mathrm{s}$. It follows that for such low velocities, higher measurement accuracy than for VISAR will be assured by the classic Michelson interferometer.

Samples with plastic deformations created by laser pulse-induced shock waves have been analyzed in view of their microstructure. Samples of austenitic stainless steel (AISI 304), irradiated in conditions similar to those assumed in the model for stronger shock waves were selected. Surface plastic deformations as well as material microstructure have been analyzed by a laser confocal microscope (Keyence VK-X100). The selected samples were analyzed also with a scanning electron microscope. All measured samples, treated with different laser pulse energy have shown durable plastic deformation in the shape of circularly symmetric imprints with a diameter of around $2.5 \div 4.5 \mathrm{~mm}$ and depth of 23 to $32 \mu \mathrm{m}$ depending on pulse energy. Figure 5 presents exemplary results of measurements for a sample deformed under a laser pulse energy of around $1.4 \mathrm{~J}$.

The analyses of material microhardness in the direction perpendicular to the surface did not show any hardness changes related to the deformation process under a shock wave. Moreover, any changes in grain size of austenite related to the thermal effect or the surface deformation were not found (Fig. 6).

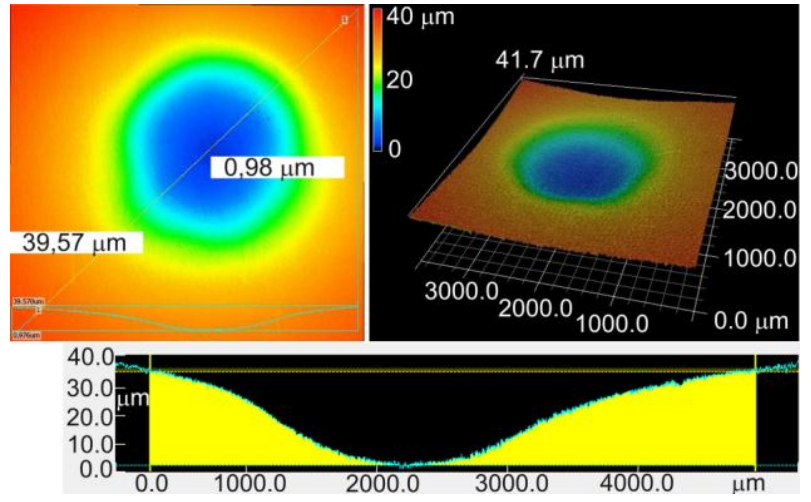

Fig. 5. Contour maps and 3D-view surface profiles for the sample deformed using laser pulse energy of around 1.4J. Numbers $0.98,39.57$ and $41.7 \mu \mathrm{m}$ inserted in the upper-left image indicate the depth measured from the bottom of the imprint.

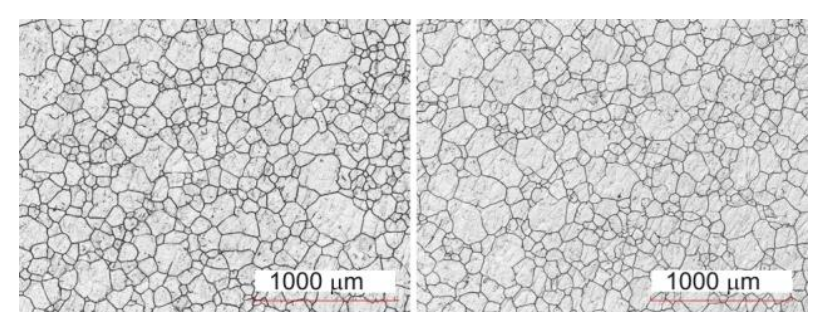

Fig. 6. Image from a laser confocal microscope of the sample (304 steel) after electrochemical etching: a) surface without deformation; b) surface after deformation under laser pulse with energy of around 1.4J.

The intended applications of the method described include analyses in micro- and nanoscale of typical and advanced functional construction materials as well as thin, hard layers.

The presented research has been conducted under the project entitled "Development of a new testing method of dynamic materials hardness, thin films and mechanical properties under a high stress rate", No 2013/09/B/ST8/03468, supported by the Polish National Science Centre (call OPUS 5).

\section{References}

[1] J. Wang, N.R. Sottos, R.L. Weaver, J. Mech. Phys. Solids 52, 999 (2004).

[2] R. Ecault et al., Composites A 53, 54 (2013).

[3] K. Jach et al., Computer modeling of dynamic interactions of bodies using free point method, Wydawnictwo Naukowe PWN, Warszawa, (2000) (in Polish).

[4] D.H. Dolan, Foundations of VISAR analysis, SANDIA REPORT, SAND2006-1950 (2006): http://www.sandia.gov/pulsedpower/prog_cap/pub_papers/061950.pdf. 